\title{
Un estudio del uso de modelos moleculares en la didáctica del enlace covalente en bachillerato
}

\author{
Esther Cascarosa Salillas. Universidad de Zaragoza \\ Francisco J. Fernández-Álvarez. Universidad de Zaragoza \\ Francisco J. Santiago. IES Pablo Serrano, Zaragoza
}

\section{Recepción: 22.06.2018 | Aceptado: 15.09.2018 \\ Correspondencia a través de ORCID: Esther Cascarosa}

0000-0002-3696-7673

Citar: Cascarosa Salillas, E., Fernandez-Alvarez, FJ. y Santiago, FJ. (2018). Un estudio del uso de modelos moleculares en la didáctica del enlace covalente en bachillerato. ReiDoCrea, 7 , 179-189.

\begin{abstract}
Resumen: Algunos de los conceptos complejos trabajados desde las asignaturas de ciencias requieren un alto nivel de abstracción para su comprensión, lo que supone una dificultad para su aprendizaje. El empleo de imágenes y modelos en 3-Dimensiones puede ser una herramienta útil para mejorar el proceso de aprendizaje de estos conceptos. En este estudio analizamos el efecto del uso de modelos moleculares en el aprendizaje del enlace covalente con alumnos de bachillerato. Se ha observado que las calificaciones obtenidas por los alumnos no se corresponden con el grado de comprensión de los conceptos, dándose el caso de que estudiantes con altas calificaciones tienen dificultades a la hora de emplear la teoría de enlace de valencia (TEV) para relacionar hibridación de orbitales atómicos con forma de las moléculas. Así mismo se ha demostrado que, tras las sesiones con modelos moleculares, los estudiantes han mejorado su capacidad de realizar representaciones geométricas de diferentes moléculas, lo que sugiere que, en el contexto analizado, el empleo de este tipo de herramienta docente en la didáctica del enlace covalente repercute positivamente en el aprendizaje.
\end{abstract}

Palabras clave: Enlace covalente | Modelos moleculares

\section{A study of the use of molecular models in the teaching of covalent-bond in high school}

\begin{abstract}
Science subjects contain complex and abstract concepts. Thus, the use of certain tools such as images or 3-D models could be useful and motivating tools to improve the teaching learning process. For this reason, this study is therefore aimed at analysing student's understanding of covalent-bond concepts after the management of molecular models to improve the understanding of the same. Student's showed great results but these were not related to a proper understanding of them but to the fact of using molecular models since students improved their geometric abilities to represent molecules. As a result, students proved the effectiveness of these aforementioned models in the teaching of the covalent-bond, in the analysed context.
\end{abstract}

Keywords: Covalent-bond | Molecular models

\section{Introducción}

\section{La inteligencia visual y la necesidad del empleo de imágenes en la didáctica de conceptos abstractos}

Munari (1990) destacó el papel fundamental de la comunicación visual en la correcta transmisión de información, evitando falsas interpretaciones, por parte del receptor. En el marco docente, hablaríamos de una comunicación visual intencional con la que se pretende que la imagen transmita un significado específico. Costa y Moles (1991) realizaron un esquema (Esquema 1) para representar la comunicación visual de carácter didáctico. En dicho esquema se muestra que es necesario que emisor (docente) y receptor (alumno) compartan un código común en referencia al concepto tratado. 


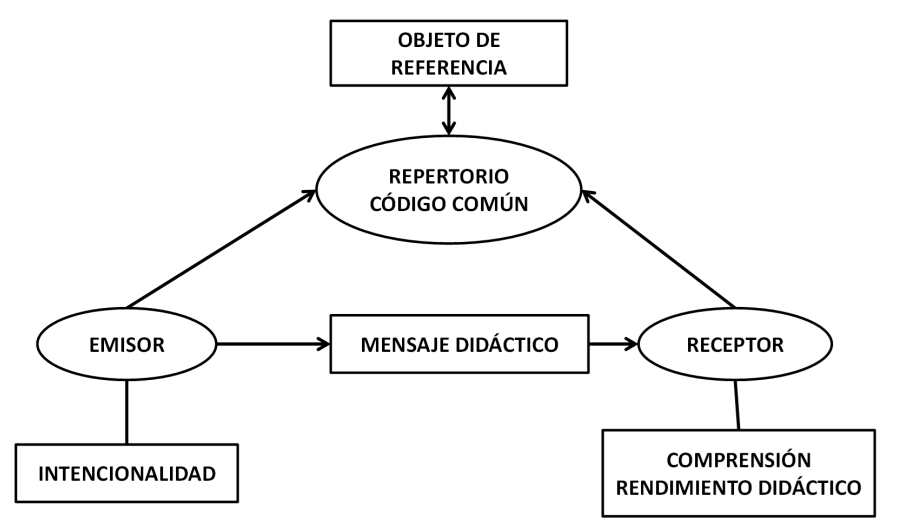

Figura 1: Diagrama del proceso de comunicación visual en el contexto docente.

En base a ello, el uso de imágenes (o en el caso de este trabajo, modelos moleculares en 3-Dimensiones) en la didáctica de la ciencia puede suponer un apoyo a la hora de establecer un código común que facilite el proceso de aprendizaje y evite errores durante el mismo.

Las teorías más frecuentemente empleadas en educación secundaria para explicar el enlace covalente son la Teoría de Lewis, la Teoría de Repulsión de Pares de Electrones de la Capa de Valencia (TRPECV) y la Teoría del Enlace de Valencia (TEV). Las teorías TRPECV y la TEV, en las que la geometría espacial juega un papel fundamental, permiten además racionalizar la forma de las moléculas. Las herramientas docentes habitualmente empleadas para abordar el aprendizaje de estos conceptos en el aula son las ilustraciones de los libros de texto, la pizarra y la destreza artística del profesor para dibujar en ella figuras con profundidad (3-D). Por tanto, la capacidad de los estudiantes para poder generar y visualizar esas figuras geométricas tridimensionales en su mente condiciona en gran medida el proceso de enseñanza del enlace covalente.

En 1998, Howard Gardner publicó la teoría de las inteligencias múltiples. Esta teoría postula la existencia de una diversidad de inteligencias (no sólo una) y señala a la inteligencia espacial como el conjunto de habilidades mentales relacionadas directamente con la navegación y la rotación de objetos en nuestra mente. Así mismo, se ha visto que arquitectos, los cuales están acostumbrados a resolver problemas donde la geometría juega un papel importante, poseen un alto nivel en este tipo de inteligencia. Por tanto, es razonable pensar que el desarrollo de la inteligencia espacial en los estudiantes podría influir favorablemente en el proceso de aprendizaje y comprensión de conceptos con alto nivel de abstracción como, por ejemplo, el enlace covalente. En ese sentido, se ha demostrado que actividades enfocadas a trabajar el desarrollo de la visión espacial con el objetivo de facilitar la comprensión de conceptos relacionados con aéreas de la ciencia, y específicamente, en conceptos donde la geometría juega un papel clave como la identificación de cristales en geología, han sido productivas (Mateo González y Martínez Peña, 2013). Así mismo, el trabajo en el desarrollo de los procesos cognitivos ha demostrado ser eficaz para mejorar las capacidades de aprendizaje (Álvarez Rodríguez, 2007).

En este contexto, es razonable pensar que el uso de modelos moleculares o figuras tridimensionales durante la explicación de conceptos con un alto nivel de abstracción puede facilitar el proceso de aprendizaje, pues permite que el estudiante relacione mediante una imagen mental la disposición de la molécula que se está estudiando con la naturaleza de los enlaces covalentes presentes en la misma. Estos modelos se 
usan habitualmente en las clases de química universitaria, pero, a pesar de las facilidades que pueden suponer para la comprensión de conceptos abstractos, apenas se usan en estudios medios.

\section{Dificultades en el proceso de enseñanza-aprendizaje del concepto del enlace covalente}

El aprendizaje y comprensión de muchos de los conceptos impartidos en la asignatura de Química presentan una gran dificultad para el alumnado. Tanto es así que son varios los estudios que han demostrado que la didáctica del enlace químico, en el que queda englobado el enlace covalente, es una de las unidades que más dificultad de comprensión presenta para el alumnado (Riboldi, Pliego y Odetti, 2004; Kim-Chwee y Treagust, 1999; De Posada, 1999).

Son varios los factores que dificultan el aprendizaje del concepto de enlace covalente, entre los que destacamos:

- Las dificultades que presenta la transposición didáctica de conceptos de la ciencia en el ámbito escolar, que, en muchos casos, otorga al docente un papel fundamental en la adquisición de contenidos (Solarte, 2006).

- El nivel de abstracción de los conceptos químicos. Así mismo, el grado de abstracción de estos mimos presenta una evolución a una mayor complejidad en el salto de los alumnos de Secundaria a Bachillerato (Pozo y Gómez-Crespo, 1998).

- La visión espacial del estudiante y del docente, que es una destreza fundamental a la hora de comprender conceptos de alto nivel de abstracción como el enlace covalente, ya que permite al estudiante representar una figura mental de la estructura molecular.

- La comprensión de los términos empleados en la didáctica de enlace covalente, tales como: hibridación, promoción electrónica, solapamiento o repulsión electrónica entre otros, están asociados con conceptos muy específicos. Por ello, es de gran importancia que el alumnado comprenda con claridad estos términos y sea capaz de realizar una asociación correcta entre ellos. Por esta razón, es importante que el docente haga un trabajo específico dedicado a la enseñanza del vocabulario empleado durante las sesiones, para que ambos, el docente y el alumnado, sean partícipes de una terminología común que posibilite la comprensión y aumente el grado de adquisición de conocimientos por parte del estudiante (Perales-López y Romero-Barriga, 2005).

Los factores descritos anteriormente ponen de manifiesto que el enlace covalente es un concepto con un alto nivel de complejidad. Sin embargo, este nivel de complejidad puede que no sea tan obvio para el alumnado, quien puede llegar a considerar la unidad didáctica del enlace químico como una de las menos complejas (Cárdenas, 2006). Este hecho puede estar asociado con el alto nivel de complejidad que los propios conceptos planteados presentan, haciendo que el alumnado no sea consciente de la magnitud y abstracción de éstos. Una consecuencia posible de lo anteriormente descrito es un bajo nivel de adquisición y comprensión de los conceptos del enlace covalente (De Posada 1999). Por lo que, el desarrollo de nuevas metodologías efectivas que mejoren el proceso de enseñanza-aprendizaje del enlace covalente es una línea de investigación de gran interés.

\section{Empleo de modelos moleculares en la didáctica del enlace covalente}


Hodson (1994) defendía que los estudiantes deben conocer los modelos y reconocerlos como productos obtenidos de la ciencia (aprender la ciencia); saber generar y evaluar sus propios modelos (aprender a hacer ciencia) y entenderlos como instrumentos con los que se propaga y difunde la ciencia (aprender acerca de la ciencia). El empleo de ejercicios dinámicos donde el alumnado pueda representar las moléculas planteadas en los problemas puede ser una buena estrategia para la mejora del proceso de enseñanza-aprendizaje del enlace covalente. Tanto es así que, estudios realizados con estudiantes de edades comprendidas entre 16 y 17 años acerca de conocimientos académicos del enlace covalente, permitieron concluir que el $23 \%$ de los alumnos no consideraban el papel de la electronegatividad de los átomos en el enlace polar; el $27 \%$ consideraba que la geometría de la molécula estaba influida por la polaridad del enlace (en este punto, habría que recalcar que la polaridad de los enlaces puede llegar a influir en el ángulo entre los enlaces, pero no en las disposición de los enlaces); y casi un $30 \%$ de ellos confundía los concepto de fuerza intermolecular y enlace químico, llegando a confundir tanto su papel dentro o fuera de la molécula, como su aplicación en redes cristalinas (Peterson y Treagust, 1989; Peterson, Treagust y Garnett, 1989). Así mismo, en un ejemplo claro de fallo en la comprensión de conceptos, Caamaño y Casassas (1987) probaron que la mayoría de los alumnos no son capaces de proporcionar una definición apropiada de orbital atómico.

Para facilitar la comprensión y aplicación de los contenidos del enlace covalente, se meditó la posibilidad de utilizar los modelos moleculares como herramienta didáctica clave en el proceso de aprendizaje. Este planteamiento estaría de acuerdo con las ideas de Lemke (2006) que defienden que el proceso de aprendizaje puede ser llevado a cabo tanto mediante el lenguaje como a través de representaciones visuales. El empleo de los modelos moleculares para representar figuras en 3-Dimensiones facilitaría el proceso de enseñanza y aprendizaje y la asimilación de conceptos de alto nivel de abstracción y complejidad, y que además resultan cruciales a la hora de entender la química y otras asignaturas del área de la ciencia como la biología (Móndelo y Martínez, 1994). Asimismo, sería interesante investigar la posibilidad de utilizar otro tipo de representaciones de moléculas, ya que como destaca Treagust et al. (2000) la variedad de formas de representar moléculas es muy amplia y que cada una de ellas resalta cualidades particulares de ellas. De ese modo, se podría mejorar la eficiencia del proceso de aprendizaje y proveer al alumnado de diferentes formas de expresar un mismo concepto.

\section{Objetivos o hipótesis}

En este contexto, decidimos llevar a cabo una investigación inicial para estudiar la influencia del empleo de modelos moleculares en el proceso de aprendizaje del enlace covalente en alumnos de $2^{\circ}$ curso de Bachillerato. En concreto, los resultados obtenidos en este estudio han servido para: i) Analizar si el empleo de modelos moleculares puede facilitar la comprensión de conceptos abstractos que requieren visión espacial; ii) Estudiar la idoneidad de los modelos moleculares como herramienta para la didáctica del enlace covalente a nivel curricular en el curso de segundo de bachillerato.

\section{Métodos}

Este estudio se ha realizado a lo largo del curso 2017-2018 con estudiantes del instituto público de enseñanza secundaria Pablo Serrano de Zaragoza. La muestra de estudiantes con los que se ha llevado a cabo esta investigación inicial es de 27 estudiantes, de los cuales 18 son chicas y 9 son chicos. 
La metodología seguida en esta investigación inicial se basa en el análisis de las respuestas dadas por los alumnos ante actividades de tipo deductivo y explicativo, propuestas tras algunas de las etapas del estudio. A continuación, se muestra la secuencia didáctica seguida y las producciones de los alumnos, analizadas en esta investigación inicial (Figura 2).
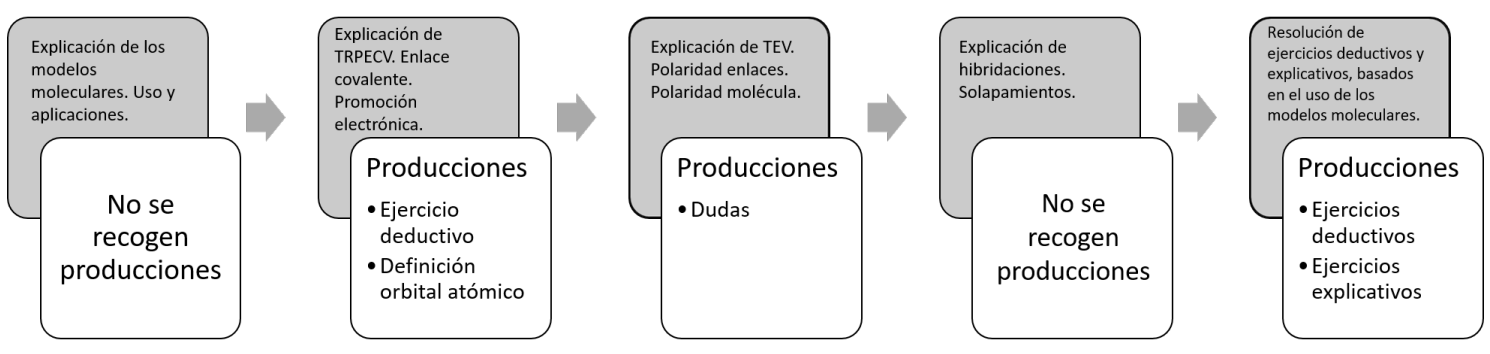

Figura 2: Secuencia didáctica y producciones de los alumnos, recogidas para su análisis.

Fase 1: En la parte inicial de este trabajo, los estudiantes recibieron una explicación detallada acerca de los modelos moleculares, de su uso y de sus aplicaciones. Tras ello, se dedicó el tiempo necesario para que los utilizaran. Una vez aprendido el uso de estos modelos, se les pidió representar estructuras cristalinas de compuestos iónicos con el objetivo de facilitar la comprensión del concepto de índice de coordinación. Seguidamente se trabajaron las dos teorías de enlace, la teoría de repulsión de pares de electrones de la capa de valencia (TRPECV) y la teoría de enlace de valencia (TEV). En cada uno de los pasos de la secuencia didáctica seguida, se utilizaron los modelos moleculares. Así mismo, durante las explicaciones de las distintas geometrías que presentaban las moléculas, se les explicó el significado de las cuñas (que simbolizan los enlaces formados en distintos planos del papel) como recurso gráfico para representar la geometría de las moléculas. Pidiendo a los estudiantes que en adelante hicieran uso de ellas en la resolución de los ejercicios.

Fase 2: Los objetivos planteados para esta segunda fase fueron, la explicación del concepto de enlace covalente y de la promoción electrónica. Posteriormente, se trabajó el concepto de promoción electrónica, el cual, explica la diferencia de valencias presentes en átomos de un mismo grupo en la tabla periódica. Para llevarlo a cabo, se utilizaron los diagramas que relacionan los orbitales y los niveles de energía. Para explicar esta relación, se les recordaron las deducciones obtenidas a través de los espectros de emisión, lo que permitió deducir los distintos orbitales presentes en los diferentes niveles de energía donde se pueden encontrar electrones en un átomo.

Antes de finalizar la sesión, se les pidió a los alumnos que realizaran un ejercicio de carácter deductivo (consideraremos como ejercicio de carácter deductivo a aquellos donde a partir de la configuración electrónica de los átomos, el alumno debe deducir la molécula que pueden formar). Este ejercicio, (anexo I), fue recogido en una cuartilla para estudiar sus respuestas. También se les pidió que, basándose en lo visto hasta ese momento, dieran una definición de orbital atómico.

Fase 3: Esta sesión constó de una explicación de la TEV, haciendo hincapié en la necesidad de la presencia de un electrón desapareado en los orbitales para poder generar un enlace covalente. Para evaluar la adquisición de conocimientos, los alumnos realizaron diferentes ejercicios donde trabajan el concepto de polaridad de la molécula dependiendo de su geometría y la polaridad de sus enlaces covalentes. Al 
finalizar esta fase, se pidió a los alumnos que expusieran las dudas que tenían, lo cual ha servido para analizar el proceso de aprendizaje del enlace covalente.

Fase 4: Esta fase presentaba una gran dificultad debido a la abstracción del concepto que se iba a tratar. En primer lugar, se explicó el concepto de hibridación. Para exponer la necesidad de la formación de enlaces híbridos, se utilizaron las ilustraciones de la geometría de los orbitales. Se explicó que la geometría y disposición espacial de los orbitales sin hibridar no explica la geometría que las moléculas adoptan. Seguidamente, se introdujeron los distintos tipos de hibridación a través de ejemplos concretos con moléculas reales. Durante la explicación de cada una de las moléculas se hizo uso de modelos moleculares. De esa manera se generaron las figuras geométricas correspondientes a cada molécula, y para se expusieron los solapamientos laterales de los orbitales $p$ no hibridados, característicos de los enlaces covalente tipo $\pi$, para justificar como las disposiciones geométricas de los orbitales $\mathrm{p}$ sin hibridar solo permiten un posible solapamiento.

Fase 5: Esta fase se diseñó con el objetivo de analizar si el uso de los modelos moleculares ayudaba a los alumnos a resolver ejercicios tanto de carácter deductivo, como de carácter explicativo, entendiendo por carácter explicativo aquellos en los que se proporciona la formula molecular de una molécula y, a partir de ahí, el alumno debe explicar las características del átomo central y las propiedades de la molécula. Estos ejercicios pueden verse en el anexo II. Se recogieron datos acerca de las respuestas dadas por los alumnos.

\section{Resultados}

\section{Ejercicio deductivo recogido en la Fase 2}

Una vez analizado los resultados obtenidos, pudimos comprobar que la mayoría de los alumnos pudieron deducir la fórmula molecular. Clasificamos las respuestas recogidas en dos grupos: aquellos que respondieron a la pregunta sin dar ninguna explicación, y en el otro los que si dieron una justificación.

No proporcionaron justificación: De los alumnos que no añadieron una explicación, dividimos las respuestas en dos grupos: los que realizaron el ejercicio a través de diagramas de orbitales y los que usaron estructura de Lewis y diagrama de orbitales.

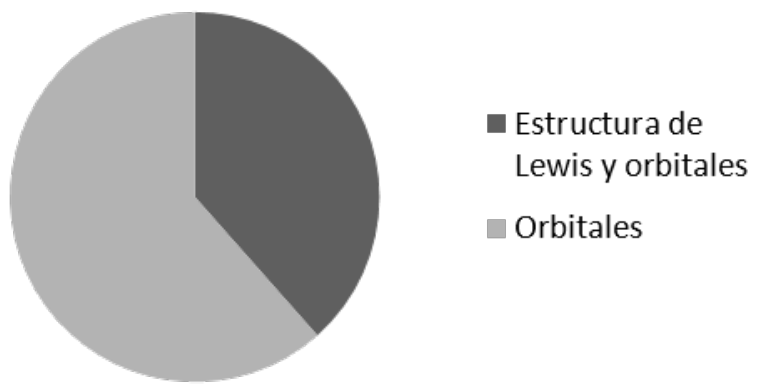

Figura 3: Representación de las opciones utilizadas por los alumnos que no dieron explicación en la resolución.

Proporcionaron justificación: Las estrategias de respuesta fueron muy similares a las vistas en el grupo anterior. Así mismo, dos alumnos utilizaron ambas estrategias en la justificación de la respuesta. 
A continuación, clasificamos las respuestas dadas por los alumnos dependiendo de su estrategia elegida:

Orbitales y estructura de Lewis: Se podrían considerar las respuestas más completas. Ambas se basaron en la necesidad de completar el octete electrónico de los átomos y en la formación de un enlace covalente a través de la compartición de electrones.

Estructura de Lewis: Dos alumnos confundieron enlace covalente llamándolo enlace sencillo. Un alumno habló del papel de la electronegatividad para formar el enlace covalente. En general, sus explicaciones se basaron en completar el octete electrónico.

Orbitales: Sus respuestas se basaron en los electrones desapareados y la representación de estos en diagrama de cajas para representar los orbitales. No explican que el enlace covalente sencillo se forma generalmente por solapamiento de orbitales semiocupados.

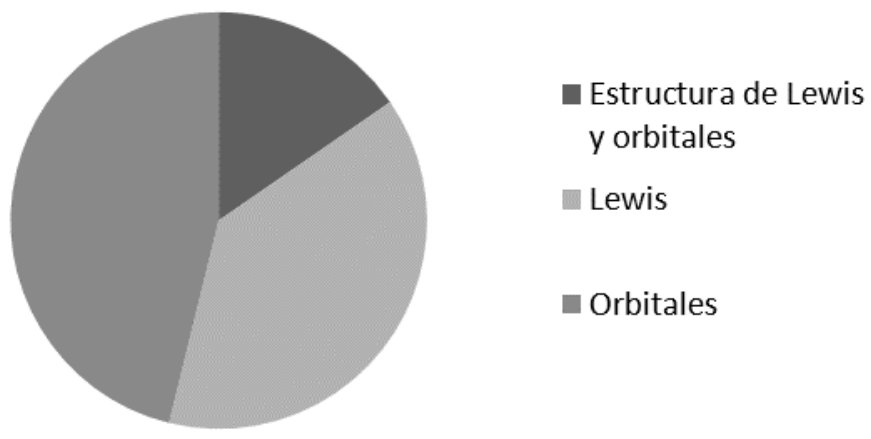

Figura 4: Representación de las opciones utilizadas por los alumnos que dieron explicación en la resolución.

Los alumnos supieron deducir la molécula más probable que podían formar los átomos propuestos. Sin embargo, las explicaciones proporcionadas no muestran una explicación teórica sólida a la deducción. Además, cabe resaltar que ninguno de ellos razonó acerca de la geometría de la molécula, aparentemente no consideraron la TRPECV. Asimismo, ninguno de ellos hizo del uso de las cuñas para dibujar la geometría de la molécula.

\section{Definición de orbital recogida en la Fase 2}

Dieciocho de los alumnos proporcionaron una definición de orbital. Se analizaron las definiciones tomando como términos clave probabilidad y región (o palabras equivalentes). En mayor o menor medida, todos relacionan el término orbital con los electrones, entendiendo esta región como la zona donde se sitúan los mismos. Sin embargo, solo seis usaban la palabra probabilidad en sus definiciones.

\section{Dudas de los alumnos recogidas en la Fase 3}

A continuación, se muestran las dudas expresadas por los alumnos en esta fase: 
- “¿Por qué no pueden promocionar los electrones del oxígeno y si los del azufre?"

- “¿La hibridación se da en moléculas concretas o es habitual?”

- “¿Entre que tipos de orbitales se producen las hibridaciones?"

- “¿Los orbitales p vacíos no afectan al enlace?"

- “¿El enlace $\sigma$ no necesita orbitales p?"

- “¿Por qué hay dos enlaces $\pi$ en el triple enlace?"

En general, las preguntas realizadas por los alumnos están dentro de lo que habitualmente se puede cuestionar un alumno de estos cursos académicos, sin embargo, resulta interesante que uno de ellos se plantee si la hibridación es algo que ocurre de manera generalizada. Esta cuestión no requiere de una aclaración de los conceptos trabajados, sino que busca una generalización, lo que supone un paso previo a la construcción del modelo global de enlace.

\section{Ejercicios deductivos y explicativos de la Fase 4}

A continuación, se muestra el análisis realizado sobre las repuestas de los alumnos a los ejercicios propuestos. En la figura 5 puede apreciarse como los alumnos utilizaron los modelos moleculares en la resolución de esos ejercicios.

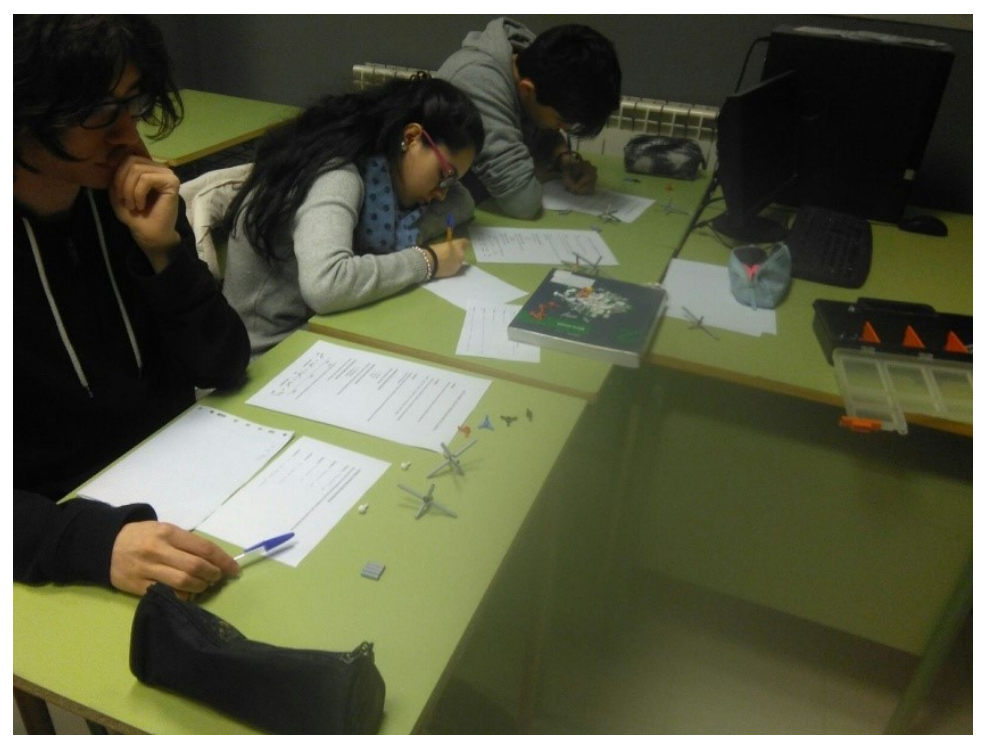

Figura 5: Imagen de los alumnos utilizando los modelos moleculares en la resolución de los ejercicios propuestos.

\section{Pregunta de carácter explicativo:}

Apartado a: Todos los alumnos escribieron correctamente la estructura de Lewis. Cabe destacar que solamente dos de los alumnos no situaron los pares de electrones no enlazantes de los átomos.

Apartado b: Todos los alumnos comenzaron el ejercicio definiendo la TRPECV. Así mismo, todos los alumnos dedujeron la estructura geométrica que presentan las dos moléculas propuestas en el ejercicio. Seis de los alumnos hicieron uso de las cuñas a la hora de hacer una representación de las moléculas. Por otro lado, cuatro alumnos dibujaron las moléculas de manera plana, sin utilizar la profundidad $y$, otros tres alumnos dedujeron la estructura geométrica de las moléculas sin dibujar las moléculas. 
Apartado c: La mayoría de los alumnos dedujeron la polaridad de las moléculas. Sin embargo, cabe destacar el dato de que seis alumnos no dibujaron, ni las moléculas, ni los momentos dipolares de los enlaces covalentes. Así mismo, otros dos alumnos emplearon la palabra vector, no introducida al trabajar estos conceptos en clase, para definir el momento dipolar.

Apartado d: Todos señalaron que los dos átomos centrales presentaban hibridación sp3. Destacar la respuesta de dos de los alumnos que relacionaba la hibridación de los orbitales y la disposición de estos en el espacio con la geometría tetraédrica de las moléculas. Sin embargo, podemos apreciar ciertos errores conceptuales en las justificaciones dadas por los alumnos. Cuatro de los alumnos relacionan el concepto de hibridación con el tipo de enlace formado entre los átomos. Así mismo, otros dos alumnos relacionaron la hibridación con la promoción electrónica para generar electrones desapareados, relacionando uno de ellos, la promoción electrónica con el solapamiento de orbitales. Otro alumno dedujo la hibridación a partir de la promoción electrónica que genera 4 orbitales con 4 electrones desapareados. Otro de los alumnos utilizó el mismo argumento, y también, se apoyó en la estructura de Lewis realizada en el apartado a.

\section{Pregunta de carácter deductivo}

En este ejercicio, se analizó el apartado "c" del ejercicio 2 (anexo II) en el cual se pide la deducción de la molécula que podían formar dos átomos a partir de sus configuraciones electrónicas. La mayoría de los alumnos se apoyaron en la estructura de Lewis y los conceptos de la promoción electrónica y la regla del octeto para deducir la fórmula molecular. De las respuestas estudiadas, 8 de ellas proporcionaban la fórmula molecular correcta. Uno de los alumnos que no proporcionaron la fórmula, aporta una explicación correcta a través de la promoción electrónica.

\section{Evolución de los alumnos en el ejercicio deductivo}

Se ha llevado a cabo un pequeño análisis de la evolución del concepto de enlace a lo largo de este trabajo. Este análisis se realizó a través del estudio de las respuestas que dos de los alumnos dieron al ejercicio recogido en la fase 1 y a la respuesta escrita en el apartado c del ejercicio 2 en la fase 4. Ambos alumnos respondieron correctamente en ambos ejercicios (fase 1 y fase 4 ). Sin embargo, mientras que uno de ellos repitió el mismo esquema de respuesta, el otro no utilizó el diagrama de Lewis, como previamente hizo en el ejercicio de la fase 1. Por lo demás, no se aprecian grandes cambios en las estrategias resolutivas de ambos alumnos.

\section{Discusión}

El proceso de enseñanza-aprendizaje del enlace covalente presenta muchas dificultades. Desde la explicación de conceptos de alto nivel de abstracción, hasta conseguir que los alumnos centren sus esfuerzos en la comprensión de los conceptos y su correcta aplicación, son muchos los frentes que el docente debe trabajar para el éxito de la adquisición de este concepto. Para ayudar a la comprensión del concepto de enlace covalente, el uso de elementos visuales se presenta como una herramienta muy útil. Este concepto, y otros relacionados con él, tales como la hibridación de los orbitales o la geometría presentada por las moléculas, pueden ser abordados a través del uso de representaciones en 3-Dimensiones, facilitando de esta manera el proceso de aprendizaje al alumnado. 
Así mismo, a la hora de experimentar las distintas posibles moléculas que los átomos pueden generar entre sí dependiendo de sus características, el aprendizaje en el uso de los modelos moleculares puede ser una estrategia interesante. Asimismo, los alumnos pueden encontrar atractivo este tipo de actividades que rompen la dinámica de clase que normalmente se lleva a cabo en los cursos de Bachillerato.

Los resultados obtenidos en este estudio preliminar muestran que introducir el uso de modelos moleculares en la etapa de bachillerato permite que los estudiantes creen una imagen mental del concepto de enlace covalente facilitando el aprendizaje de este.

Una prueba de que el empleo de modelos como herramienta docente supone un paso "facilitador" de estas representaciones, es que los alumnos han realizado dibujos basados en el uso de estos modelos, para representar la geometría espacial de las moléculas incluidas en los ejercicios propuestos. Por otro lado, se pudo apreciar una mejora en la resolución de ejercicios de tipo deductivo.

Sin embargo, en muchas de las respuestas dadas por los alumnos se identifican errores conceptuales y falta de argumentos teóricos que justifiquen sus respuestas. Por ejemplo, varios alumnos dedujeron la polaridad de una molécula sin dibujar su geometría y sin utilizar los momentos dipolares de los enlaces covalentes de la misma.

Por tanto, podemos concluir que este estudio indica que el empleo de modelos moleculares facilita el proceso enseñanza/aprendizaje de conceptos químicos complejos como son: enlace covalente, hibridación y forma de las moléculas. Lo que nos anima a continuar con nuestras investigaciones en este campo.

\section{Agradecimientos}

Los autores agradecen al proyecto MINECO EDU2016-76743-P su apoyo en el desarrollo de este trabajo.

\section{Referencias}

Álvarez Rodríguez, S. (2007). Procesos cognitivos de visualización espacial y aprendizaje. Revista de Investigación en Educación, 4, 61-71.

Caamaño, A. y Casassas, E. (1987). La comprensión de la estructura de la materia y del cambio químico en estudiantes de 15 y 16 años. Enseñanza de las Ciencias, 159-160.

Cárdenas, S. F. A. (2006). Dificultades de aprendizaje en química, caracterización y búsqueda de alternativas para superarla: Ampliación y continuación.: Ciência \& Educacão, 3(12), 333346.

Costa, J. y Moles, A. (1991). Imagen didáctica. Barcelona: Ceac.

De Posada, J. M. (1999). Concepciones de los alumnos sobre el enlace químico antes, durante y después de la enseñanza formal. Problemas de aprendizaje. Enseñanza de las Ciencias, 17(2), 227-245.

Gardner, H. (1998). A Reply to Perry D. Klein's 'Multiplying the problems of intelligence by eight. Canadian Journal of Education, 23(1), 96-102.
Hodson, D. (1994). Hacia un enfoque más crítico del trabajo de laboratorio. Investigación y experiencias didácticas, 12(3), 299-313.

Kim-Chwee, D. T. y Treagust, D. F. (1999). Evaluating students understanding of chemical bounding. School Science Review, 81(294), 75-83.

Lemke, J. L. (2006). Investigar para el futuro de la educación científica: nuevas formas de aprender, nuevas formas de vivir. Enseñanza de las Ciencias, 24(1), 5-12.

Mateo González, E. y Martínez Peña, M. B. (2013). ¿Tengo Visión espacial? Simetría de modelos cristalográficos sobre alumnos de altas capacidades. IX Congreso Internacional sobre investigación en didáctica de las ciencias. Girona.

Móndelo, M. y Martínez, C. (1994). Materia inerte o materia viva ¿Tienen ambas constitución atómica? Enseñanza de las Ciencias, 12(2), 226-233

Munari, B. (2a ed.) (1990). Diseño y comunicación visual. Barcelona: Gustavo Gili, S.L. 
Perales-López, JC., y Romero-Barriga, JF. (2005). Procesamiento conjunto de lenguaje e imágenes en contextos didácticos: Una aproximación cognitiva. Anales de Psicología, 24(1), 1330 .

Peterson, R. F. y Treagust, D. F. (1989). Grade-12 students'" misconceptions of covalent bonding and structure. Journal of Chemical Education, 66(6), 459-460

Peterson, RF., Treagust, DF., \& Garnett, P. (1989). Development and application of a diagnostic instrument to evaluate grade 11 and 12 students' concepts of covalent bonding and structure following a course of instruction. Journal of research in Science Teaching, 26(4), 301-314.

Pozo, J. I. y Gómez-Crespo, M. A. (Ed. rev.). (1998). Aprender y enseñar ciencia. Madrid: Morata.

Riboldi, L., Pliego, O. y Odetti, H. (2004). El enlace químico: una conceptualización poco comprendida. Enseñanza de las Ciencias, 22(2), 195-212.

Solarte, M. C. (2006). Los conceptos científicos presentados en los textos escolares: son consecuencia de la transposición didáctica. Revista ieRed, 1(4), 1.

Treagust, D., Duit, R. y Nieswandt, M. (2000). Sources of student's difficulties in learning Chemistry. Revista de Educación Química, 11(2), 228-235.

\section{Anexos}

\section{Anexo I}

\section{Enunciado del ejercicio recogido durante la primera fase}

Ejercicio: Sean dos elementos A y B de configuraciones electrónicas respectivas 1s2 2s2 2p4 y 1s2 2s2 2p5. Indica y razona cuál de las siguientes propuestas es más probable para el compuesto formado entre $A$ y $B: A B 2, A 2 B, A 2 B 3$ o no reaccionan. Si forman algún compuesto, indica el tipo de enlace entre A y $B$. Razónalo.

\section{Anexo II}

\section{Ejercicio Explicativo}

1. Para las moléculas $\mathrm{SiF} 4$ y $\mathrm{CH} 3 \mathrm{Cl}$ :

a. Escribe las estructuras de Lewis.

b. Determina la geometría molecular utilizando la teoría de repulsión de pares de electrones de la capa de valencia.

c. Indica, justificando brevemente la respuesta, si se trata de moléculas polares.

d. Indica, justificando brevemente la respuesta, si alguno de los átomos implicados en estas moléculas ha sufrido alguna hibridación, indicando en su caso cuál.

\section{Ejercicio Deductivo}

2. Los elementos $A, B, y$ C tienen números atómicos 9,14 y 26 respectivamente.

a. ¿Cuál de ellos es un elemento de transición y de que elemento se trata? Razona la respuesta.

b. ¿Cuál es el elemento más electronegativo de los tres? Razona la respuesta.

c. ¿Qué fórmula y qué tipo de enlace tendrá el compuesto más probable formado por A y B? Razona la respuesta. 